\title{
Cellular and Circuitry Bases of Autism: Lessons Learned from the Temporospatial Manipulation of Autism Genes in the Brain
}

\author{
Samuel W. Hulbert ${ }^{1} \cdot$ Yong-hui Jiang $^{1,2,3}$
}

Received: 10 January 2017/ Accepted: 12 February 2017/Published online: 7 March 2017

(C) The Author(s) 2017. This article is published with open access at Springerlink.com

\begin{abstract}
Transgenic mice carrying mutations that cause Autism Spectrum Disorders (ASDs) continue to be valuable for determining the molecular underpinnings of the disorders. Recently, researchers have taken advantage of such models combined with Cre-loxP and similar systems to manipulate gene expression over space and time. Thus, a clearer picture is starting to emerge of the cell types, circuits, brain regions, and developmental time periods underlying ASDs. ASD-causing mutations have been restricted to or rescued specifically in excitatory or inhibitory neurons, different neurotransmitter systems, and cells specific to the forebrain or cerebellum. In addition, mutations have been induced or corrected in adult mice, providing some evidence for the plasticity and reversibility of core ASD symptoms. The limited availability of Cre lines that are highly specific to certain cell types or time periods provides a challenge to determining the cellular and circuitry bases of autism, but other technological advances may eventually overcome this obstacle.
\end{abstract}

Keywords Autism - Mouse models $\cdot$ Behavior $\cdot$ Cre-loxP . Cerebellum $\cdot$ Critical period

Yong-hui Jiang

yong-hui.jiang@duke.edu

1 Department of Neurobiology, School of Medicine, Duke University, Durham, NC 27710, USA

2 Department of Pediatrics, School of Medicine, Duke University, Durham, NC 27710, USA

3 Program in Genetics and Genomics, School of Medicine, Duke University, Durham, NC 27710, USA

\section{Introduction}

The neurobiological basis of Autism Spectrum Disorders (ASDs) has been a growing area of research during the past few decades. While substantial progress has been made to uncover the molecular underpinnings of the disorders, the cell types and circuits underlying autistic behaviors remain largely unknown [1]. This knowledge, however, is critical to developing targeted therapy. Neuroimaging in human patients has provided correlates between the function of certain brain regions and behavior [2, 3]. Such studies are limited by the phenotypic heterogeneity that is characteristic of ASDs, the individuals available for study, the low resolution of imaging techniques, and the inability to manipulate molecules and circuits in the human brain. For these reasons, it is technically challenging to establish causality between circuits and behavior in studies involving humans. However, animal models allow researchers to determine causation because the genetic background and environmental factors can be controlled. Furthermore, the use of laboratory rodents allows for more invasive studies that would not be feasible or ethical in humans.

Transgenic mice with ASD-causing mutations that are present in the germline have provided clues to the molecular underpinnings of the disorders, and some degree of overlap is seen across multiple mouse models [4]. However, germline, or conventional, mutant mice do not provide enough evidence to causally link a particular brain region or circuit to ASD-like behaviors. More recently, several groups have taken advantage of tools that allow for the manipulation of genes across space and time in animal models to begin dissecting the cell types, brain regions, and associated circuits contributing to ASD phenotypes. In this review, we have focused on studies that utilize conditional gene-expression technology combined with mouse models 
of ASD that have high construct validity (i.e. the molecular consequences of the mutations mimic those observed in humans) and report phenotypes that have strong face validity (i.e. appear to mimic the behavioral features of the disorders) for the core symptoms of ASDs, impaired social communication and repetitive behaviors.

\section{Assays for ASD-Related Behaviors}

Currently, ASDs are diagnosed purely on the assessment of behavioral features [5]. Therefore, behavioral analysis is an important part of the strategy to model human mutations and understand the cellular and circuitry bases of the disorders. To help achieve this goal, a number of assays that test behaviors resembling the core symptoms of ASDs have been developed for laboratory mice [6, 7]. The behaviors mentioned in this review are briefly described here and are summarized in Table 1.

\section{Tests to Assess Social Communication}

The three chamber test consists of two phases. The first phase has a caged stranger mouse in one of the chambers, a novel object in the opposing chamber, and a neutral central chamber [8]. The second phase has the caged mouse from the first phase in one chamber, a neutral central chamber, and a novel caged mouse in the opposing chamber. The first phase is considered a test for "sociability" where wildtype mice tend to spend more time in the chamber with the caged stranger mouse than in either other chamber. The second phase is a test for "preference for social novelty" or "social preference," where most wild-type mice spend more time with the novel stranger mouse than with the familiar stranger mouse or in the neutral chamber.

The habituation/dishabituation test involves introducing the test mouse to the same contained stimulus mouse over several days and then introducing a novel contained stimulus mouse [9]. Wild-type mice tend to spend less time engaging with the familiar mouse over time, but have increased interactions with the novel mouse. This is a measure of social recognition.

In the partition test, the test mouse and a stimulus mouse are in an arena on opposite sides of a clear acrylic barrier with holes $[10,11]$. The amount of time the test mouse spends near the partition is a measure of social behavior.

In direct social interaction tests, the test mouse and stimulus mouse are allowed to freely interact. The type, duration, and amount of contacts are scored [12]. One variant of this test is resident-intruder, where the stranger mouse is introduced into the test mouse's home cage [13].

Ultrasonic vocalizations (USVs) can be recorded in pups ( $\sim$ postnatal days 3-12) and in adults [14]. For pups, this simply involves briefly separating the pups from their mother. For adults, the most common test involves exposing male mice to females in estrous or their urine.

Table 1 Summary of behavioral assays.

\begin{tabular}{|c|c|c|}
\hline Assay & Resemblance to core feature of ASDs & Quantitative measurements \\
\hline Three chamber test & Social communication deficits & $\begin{array}{l}\text { Phase 1: "Sociability" - preference for social stimulus (stranger } \\
\text { mouse) over an inanimate object } \\
\text { Phase 2: "Social novelty" - preference for an unfamiliar mouse } \\
\text { over the stranger mouse used in the sociability phase }\end{array}$ \\
\hline Habituation/dishabituation & Social communication deficits & $\begin{array}{l}\text { Time spent interacting with the same mouse during the trial period } \\
\text { over several days and time spent with a novel mouse on the last } \\
\text { trial }\end{array}$ \\
\hline Partition test & Social communication deficits & $\begin{array}{l}\text { Time spent near a barrier that separates the test mouse from a } \\
\text { stranger mouse }\end{array}$ \\
\hline Direct social interaction tests & Social communication deficits & Duration, type (e.g. aggressive), and amount of social interactions \\
\hline Ultrasonic vocalizations & Social communication deficits & Number, duration, and complexity of vocalizations \\
\hline Nest-building & Social communication deficits & $\begin{array}{l}\text { Amount of nesting material used, size of nest, or numerical scoring } \\
\text { system }\end{array}$ \\
\hline Self-grooming & Repetitive behavior & Time spent grooming or number of grooming bouts \\
\hline Jumping & Repetitive behavior & Time spent jumping or number of jumping bouts \\
\hline Digging & Repetitive behavior & Time spent digging or number of digging bouts \\
\hline Hole board test & Repetitive behavior & $\begin{array}{l}\text { Number of total hole-pokes or number of consecutive }(2+) \text { pokes } \\
\text { in the same hole }\end{array}$ \\
\hline Marble-burying test & Repetitive behavior & Number of marbles buried \\
\hline
\end{tabular}


The number, duration, and complexity of USVs can be compared.

Nest-building is considered to be another measure of social behavior. The test mouse is given a small amount of cotton or other material to build a nest in its home cage. The amount of material used, the size of the nest, or a scoring system can be used to compare experimental groups [15].

\section{Tests to Assess Repetitive Behavior}

Spontaneous repetitive behaviors include excessive selfgrooming, jumping, or digging [16]. The time spent engaging in each behavior or number of bouts can be compared.

The hole board test also measures repetitive behavior. Mice are placed on an apparatus with circular holes arranged in a grid [17]. Mice that display repetitive behavior have more nose-pokes into the holes, or explore the same hole repeatedly.

Finally, in the marble burying test, the test mouse is placed in a clean cage with marbles positioned on top of bedding [18]. Mice with repetitive behavior bury more marbles in the bedding than their wild-type littermates.

\section{Tools to Manipulate Gene Expression}

The Cre-loxP system is the approach taken most often to manipulate gene expression in a cell-type-specific or timedependent manner [19]. For cell-type-specific manipulations, this method typically involves breeding mice that contain the Cre recombinase transgene under the control of a specific promoter with mice that have the target gene flanked by loxP sequences (floxed) [20]. Deletions, and rarely duplications, of the target DNA occur when the lox $P$ sequences are oriented in the same direction in either the cis or trans configuration, whereas inversions occur if the loxP sequences are oriented in opposite directions. For more precisely-timed manipulations, the Cre recombinase protein is fused to a mutated estrogen receptor, so that tamoxifen treatment causes translocation into the nucleus and subsequent site-specific recombination [21]. Other, but less common, methods for inducible gene expression include the Tet-Off (tTA) and Tet-On (rtTA) systems, where doxycycline treatment represses and activates transcription, respectively [22].

While extraordinarily useful for temporospatial manipulation of genes in mice, the Cre-loxP system is not without its limitations. The major limiting factor is the availability of Cre lines that meet the needs of an experiment, in terms of timing and spatial distribution of Cre expression. The specificity and efficiency of recombination relies on the promoter, which may be expressed at various levels in off-target tissues or cell-types. One way to circumvent this problem is to deliver a lentivirus or adenoassociated virus construct containing Cre to mice with the target gene floxed [23]. While this method may limit the expression of Cre to a particular brain region, it is more difficult to control the expression of Cre in different cell types within a particular region. Another potential problem is that Cre expression itself can induce a phenotype by causing recombination at cryptic loxP sites in the mouse genome [24], or by affecting the expression of surrounding genes, depending on the insertion site of the transgene. Therefore it is important that proper controls are implemented to compare the phenotypes.

New advances in gene-editing technologies may eventually overcome the limitations of the Cre-loxP and similar systems. For example, it has been demonstrated that CRISPR/ Cas 9 can be used to edit the genome of adult mice and rescue disease phenotypes [25]. Moreover, methods are being developed to increase the cell-type specificity of CRISPR/ Cas9-mediated mutations [26]. However, off-target mutations using this system remain a concern and must be assessed individually [27]. Thus, some refinement of this newer technology is necessary before using it to answer questions about the neuroanatomical bases of ASDs.

\section{The Roles of Excitatory and Inhibitory Neurons in ASDs}

The two major classes of neurons in the brain are excitatory neurons, those which depolarize the neurons they project onto and thus make them more likely to produce action potentials, and inhibitory neurons, those which hyperpolarize their outputs and make them less likely to produce action potentials. It has been proposed that a common mechanism underlying ASDs is an imbalance between inhibitory and excitatory synaptic transmission in particular circuits. This may reflect a relative increase of function in excitatory neurons compared to that of inhibitory neurons [28], or vice versa. Although support for this hypothesis is currently somewhat limited, studies have begun to address it.

One important question is whether disrupting the function of either major class of neurons is sufficient to produce phenotypes related to ASDs and, if so, which types of neurons within these major classes contribute to the phenotypes. Another important question is whether different ASD-causing mutations affect the same neuronal types and circuits, or have shared circuit-level mechanisms. This may translate into whether patients with different mutations require individualized therapy in the clinic. Fortunately, the advent of conditional gene expression technologies and the 
production of multiple mouse models of ASDs with high construct validity have made it possible to start exploring these questions.

\section{Manipulations in Large Groups of Excitatory (Glutamatergic) Neurons}

Most of the published work on conditional gene expression for ASDs involves manipulation of the mouse version of the genes that are mutated in syndromic autism, such as Rett syndrome (MECP2), tuberous sclerosis complex (TSC1 or TSC2), PTEN-related disorders, and Angelman syndrome $(U B E 3 A)$. One group found that restricting the deletion of Mecp2 (loxP/y) to excitatory neurons in the forebrain postnatally, starting around postnatal day 21 (P21), with $\operatorname{Tg}($ Camk2a-cre) $93 \mathrm{Kln} \quad$ (CamKII-cre93; EMMA 01137) results in mice that display reduced sociability in a test similar to the first phase of the three chamber test and reduced preference for the novel mouse in the habituation-dishabituation test [29]. However, another group used $\mathrm{Emx} 1^{\mathrm{tm} 1(\mathrm{cre}) \mathrm{Krj}}$ (Emx1-cre; JAX 005628) to delete Mecp2 (loxP/y) in forebrain excitatory neurons and glia embryonically, starting around embryonic day 9.5 (E9.5), and found no significant changes in either sociability or preference for social novelty in the three chamber test [30]. Neither study reported any repetitive behaviors. Deletion of Mecp2 (loxP/y) selectively in glutamatergic neurons, the most abundant neurotransmitter class of excitatory neurons, in embryos with Slc17a6 ${ }^{\text {tm2(cre)Lowl }}$ (Vglut2-cre; JAX 028863) does not impact social behavior in the partition test or nose-pokes on the hole board [31]. However, selectively expressing Mecp2 (loxP-stop/y) in glutamatergic neurons with the same Cre line rescues a hypersocial phenotype on the partition test and repetitive nose-pokes on the hole board that occur in the global knockout, which suggests that restoring function to glutamatergic neurons may have network effects that subsequently increase the function of other excitatory neurotransmitters or inhibitory neurons as well [31].

On the other hand, mice that carry a deletion of another ASD candidate gene, Mef2c (loxP/loxP) with Emx1-cre spend less time interacting with the social stimulus in the first phase of the three chamber test, emit fewer USVs as pups and adults, have lower nest-building scores, and display increased repetitive jumping and fine motor movements [32]. Similarly, deletion of Cc2dla (loxP/loxP) with $\mathrm{Tg}(\mathrm{CamK2a}$-cre)T29-1Stl (CamKII $\alpha$-cre T29-1; JAX 005359) results in mice that display reduced sociability in the first phase of the three chamber test, reduced social approach in a direct social interaction test, reduced numbers of adult USVs, and increased repetitive self-grooming (but no changes in marble-burying) [33]. Mice with $T s c 1$ (loxP/loxP) deleted with CamKII $\alpha$-cre T29-1 also display reduced sociability in the three chamber test and increased marble-burying behavior [34].

Together, these findings indicate that disrupting the function of excitatory neurons can be sufficient to produce ASD-like behaviors, but conflicting findings between models indicate that manipulating one gene may not be generalizable to all ASDs. Therefore, studies using conditional gene expression of other ASD candidate genes are necessary to obtain a more complete understanding.

\section{Inhibitory (GABAergic) Neurons}

Although there is some evidence that the dysfunction of excitatory neurons contributes to ASD-like phenotypes, there is accumulating evidence for a role of inhibitory neurons as well. Using the line $\mathrm{Tg}(\mathrm{Slc} 32 \mathrm{a} 1-\mathrm{cre}) 2.1 \mathrm{Hzo}$ (Viaat-cre; JAX 017535) to delete Mecp2 (loxP/y) specifically in GABAergic cells in embryos results in mice that show nearly the full spectrum of phenotypes observed in the conventional knockout, including repetitive forelimb stereotypies, increased grooming, sequential head-pokes on the hole board task, decreased nest-building, and increased sociability in the partition test and the three chamber test [35]. Accordingly, selectively restoring Mecp2 (loxP-stop/y and loxP-stop/+) to GABAergic cells with Viaat-cre rescues the hypersocial phenotype in the partition test and nest-building deficits in both male and female mice (but repetitive behaviors were not reported) [36]. Moreover, deleting Mecp2 (loxP/y) in a subset of GABAergic cells in the forebrain, embryonically, with $\operatorname{Tg}($ Dlx6a-cre $) 1$ Mekk (Dlx5/6-cre; JAX 008199) recapitulates the nest-building, hole board, and hypersocial phenotypes, but not grooming, indicating that some features of ASD may involve other brain regions or cell types [35].

Two of the major subclasses of GABAergic neurons are parvalbumin-positive $(\mathrm{PV}+)$ and somatostatin-positive (SOM+) neurons [37]. Thus, one important question is whether either or both of these cell types contribute to ASD phenotypes. Mice that lack Mecp2 (loxP/y) in PV+ neurons, embryonically, created by crossing the floxed mice with Pvalb ${ }^{\text {tml(cre)Arbr }}$ (PV-cre; JAX 008069), display a hypersocial phenotype on the partition test, but show no differences on the hole board [38]. In contrast, mice that lack Mecp2 (loxP/y) in SOM+ neurons, embryonically, created by crossing floxed mice with $\mathrm{Sst}^{\mathrm{tm} 2.1(\mathrm{cre}) \mathrm{Zjh}}$ (SOMcre; JAX 013044), display repetitive nose-pokes on the hole board, but have no social phenotype [38]. A different study found no differences in sociability in mice lacking Mecp2 in PV+ neurons, using the same PV-cre [39]. 
However, another study confirmed that dysfunctional PV+ neurons contribute to social phenotypes whereas SOM+ neurons do not by heterozygous deletion of the mouse version of the gene underlying Dravet syndrome, Scnla $($ lox $P /+)$, in either type of neuron using PV-cre and SOMcre, respectively [40]. In this case, the mice lacking Scnla in PV + neurons show decreased sociability in the three chamber test [40].

It is likely, given that the conditional knockouts restricting Mecp2 deletion to GABAergic neurons almost completely recapitulate all of the phenotypes observed in the conventional knockout, that the pathogenesis of Rett syndrome primarily involves dysfunctional inhibitory neural networks. Some behavioral deficits have also been reported in conditional Mecp 2 knockouts where the deletion is limited to excitatory neurons, but the decrease in social behavior is distinct from the global knockout, which shows increased social interest in the partition test. It is less clear at this time whether this is generalizable to other ASDs. Therefore, more research is needed that uses conditional gene expression technology and highly penetrant ASD-causing mutations.

\section{Other Neurotransmitter Systems}

Other neurotransmitters can either be excitatory or inhibitory, depending on the action of the postsynaptic receptors that are expressed. Since the majority of neurons in the brain are either glutamatergic or GABAergic, less is known about the roles of other neurotransmitters in ASD models. However, some groups have started dissecting the roles of dopamine, serotonin, acetylcholine, and oxytocin.

Restricting the deletion of Mecp2 (loxP/y) to serotonergic neurons, embryonically, with $\mathrm{Tg}$ (Fev-cre)1Esd (PET$1 \mathrm{cre}$; JAX 012712) results in mice that display a hypersocial phenotype on the partition test as well as increased aggression during the resident-intruder test [41]. On the other hand, selectively deleting Mecp2 (loxP/y) in dopaminergic neurons, embryonically, with $\mathrm{Th}^{\mathrm{tm} 1 \text { (cre)Te }}$ (TH-cre; EMMA 00254) does not influence social behavior in these assays [41]. The PET-1 cre knockout does not have repetitive grooming or marble-burying, but these behaviors were not assessed in the TH-cre knockout [41]. This study, along with others, suggests that dysfunctional serotonergic neurons may contribute to the core symptoms of ASDs, whereas dopaminergic neurons are implicated in comorbid motor functions [42-44]. More support for the role of serotonergic neurons in ASD behaviors comes from deleting Tscl (loxP/loxP) selectively in serotonergic neurons, embryonically, with $\mathrm{Tg}$ (Slc6a4-cre)ET33Gsat (S1c6a4-cre; MMRRC 031028-UCD), which results in mice with decreased sociability in the first phase of the three chamber test and increased repetitive marble-burying behaviors [34].

Recently, the cholinergic system has also been implicated in the pathogenesis of ASD with conditional gene expression in mice. Selectively deleting Mecp $2(\operatorname{loxP} / y)$ in cholinergic neurons, embryonically, with Chat ${ }^{\text {tm2(cre)Lowl }}$ (Chat-cre; JAX 006410) produces mice that fail to show a preference for social novelty in the three chamber test, show decreased social investigation in a direct interaction test, and have impaired nest-building [45]. Interestingly, reexpression of Mecp2 (via microinjection of an AAV) in the basal forebrain, but not in the caudate-putamen, of adult mice rescues the social deficit in the three chamber test [45]. Again, this phenotype is distinct from that in the global Mecp2 knockout, but nonetheless provides insight into circuits underlying ASDs.

So far, the role of oxytocinergic neurons is less clear. Neither heterozygous (lox $P /+$ ) nor homozygous (loxP/ loxP) deletion of Pten in oxytocinergic neurons, embryonically, with Oxt ${ }^{\text {tm1.1(cre)Dolsn }}$ (Oxt-cre; JAX 024234) results in deficits in the three chamber, habituation/dishabituation, or marble-burying tests [46]. This is somewhat puzzling, given that Pten haploinsufficient, oxytocin knockout, and oxytocin receptor knockout mice show some overlapping impairments on these assays [47-50]. Perhaps mutations in PTEN and other ASD-causing mutations have more of an effect on neurons expressing the oxytocin receptor, rather than on oxytocinergic neurons. More work needs to be done to clarify the role of oxytocin and other neurotransmitters in ASDs.

\section{The Role of the Cerebellum in ASDs}

In recent years, the cerebellum has emerged as a brain region that may be implicated not only in motor impairments, but also in the core behaviors of ASDs, particularly because cerebellar Purkinje cells project to the thalamus and, for example, affect dopamine efflux in the prefrontal cortex [51]. Mice with heterozygous (lox $P /+)$ or homozygous (loxP/loxP) deletion of Tscl mostly restricted to Purkinje cells, postnatally, by means of $\mathrm{Tg}$ (Pcp2-cre)2Mpin (L7-cre; JAX 004146) fail to demonstrate preference for a social stimulus over a nonsocial stimulus or for novel social interactions over familiar ones in the three chamber test [52]. These mice also engage in elevated rates of self-grooming [52]. Similarly, mice with $T s c 2$ homozygously deleted in Purkinje cells with L7-cre and heterozygously deleted in all other cell types (loxP/-) have impaired social interactions and increased rates of marble-burying [53]. Mice with Pten deleted (loxP/loxP) in Purkinje cells by L7-Cre display reduced sociability and engage in repetitive 
upright scrabbling, but have reduced self-grooming [54]. Interestingly, deleting Pten $(\operatorname{loxP} / \operatorname{lox} P)$ primarily in cerebellar granule cells with $\operatorname{Tg}$ (Gfap-cre)1Sbk (Gfap-cre) results in mice that are hyposocial in the partition test and show reduced sociability in the three chamber test, but show reduced repetitive behaviors in the marble-burying and hole board tests, and have no changes in USVs as pups [55].

Most recently, two similar studies produced opposite conclusions regarding the cerebellum's role in social behavior. Purkinje cell deletion of exon 7 of Shank2 (loxP/ loxP) with L7-Cre resulted in mice that failed to demonstrate a preference for sociability or for social novelty [56]. However, mice with a deletion of exons 6-7 of Shank2 (loxP/loxP) with $\mathrm{Tg}$ (Pcp2-cre)3555Jdhu (Pcp2-cre; JAX 010536) were similar to controls on the three chamber task and had similar USVs, but had increased nose-pokes on the hole board [57]. Both groups of mice showed normal levels of self-grooming and marble-burying.

The divergent findings regarding social behavior between the two studies using Shank 2 conditional mutants have several possible explanations. One is that minor differences in the exonic deletions could disrupt the expression of different sets of Shank2 isoforms, but current knowledge regarding the structure of Shank2 suggests this unlikely to be the case [58]. Another possibility is that the use of different Cre lines (JAX 004146 vs. JAX 010536) in the two studies may have some effect on behavior; although both Cre lines involve the same promoter, there may be differences in the spatial pattern and timing of Cre expression. The most likely explanation is that the two labs used different methods for determining behavioral phenotypes (for example, strains chosen for the stranger mice and habituation times). This last explanation is especially convincing because the wild-types in one study showed a preference for social novelty [56], whereas those in the other study did not [57], most likely indicating differences in behavioral methods.

Further investigation into the cerebellum's contributions to ASD phenotypes is clearly warranted. Moreover, the contradictory findings from similar studies underscore the need for stricter standards for behavioral methods or more side-by-side comparisons between lines of mice within investigating laboratories to make the strongest conclusions. It is unclear at this time whether, for instance, Tscl, Tsc2, Pten, and Shank2 deletions in the cerebellum have different effects on self-grooming, or whether the discrepancies between the studies are due to varying methods. It is even less clear which cerebellum-related circuitry is responsible for the ASD-like behaviors and whether there is any translational value of these findings for human patients.

\section{Developmental Time Points Implicated through Inducible Mutations and Rescues}

Clinically, ASDs are classified as a group of neurodevelopmental disorders. However, the developmental origin of ASDs has not been clearly defined. This knowledge is likely critical for effective clinical intervention. Conditional gene expression, through inducible mutations and rescues, allows researchers to determine whether ASDs are the result of disrupted development and/or ongoing neuronal dysfunction. Conditional-rescue mice also provide proof of principle for the potential of gene therapy. One attractive hypothesis for the origin of ASDs is that perturbations to synaptic development prevent patients from developing skills during a limited window of opportunity, or a critical period [59]. This would suggest that reversing the symptoms of ASD may be difficult, if not impossible. Some studies suggest that early intervention is necessary for preventing the onset of ASD-like phenotypes. However, multiple studies have also challenged the notion that ASDs represent an irreversible disruption of brain development.

Deleting Mecp2 in adulthood (loxP/y) using the ubiquitously-expressed, inducible Cre line, $\mathrm{Tg}$ (CAG-cre/Esr1*)5Amc (CAGGS-CreER; JAX 004453) and tamoxifen administration, causes the impaired nest-building phenotype that is observed in germline knockouts [60]. Reactivating Mecp2 in male (loxP-stop/y) and female (loxP-stop/ $+)$ model mice with the same Cre line and tamoxifen administration partially and completely rescue this nestbuilding impairment, respectively [61]. Neither of these studies reported any other ASD-related behaviors, nor did two other studies that reactivated Mecp2 expression $[62,63]$. However, reinstating Ube3a (loxP-stop/p+) using the same inducible Cre line fails to rescue impaired nestbuilding in these mice, even when tamoxifen is administered to newborn mice; only embryonic reinstatement of Ube $3 a$ successfully rescues this phenotype [64].

On the other hand, several ASD-related behaviors are rescued by repressing the expression of a dominant-negative form of Nrxn1 $\beta$ in excitatory neurons in adulthood using Tg(Camk2a-tTA)1Mmay (CaMKII-tTA; JAX 003010) with the administration of doxycycline, which reverses increased self-grooming and impaired sociability and preference for social novelty in the three chamber test [65]. Similarly, re-expressing normal Shank3 isoforms by reverting an inverted portion of the gene after administering tamoxifen to adult mice (loxP/loxP; CAGGS-CreER) rescues increased grooming, impaired sociability in the first phase of the three chamber test, and reduced preference for social novelty in the second phase of this test [66].

While in general these studies provide some promising evidence for the reversibility of the core symptoms of 


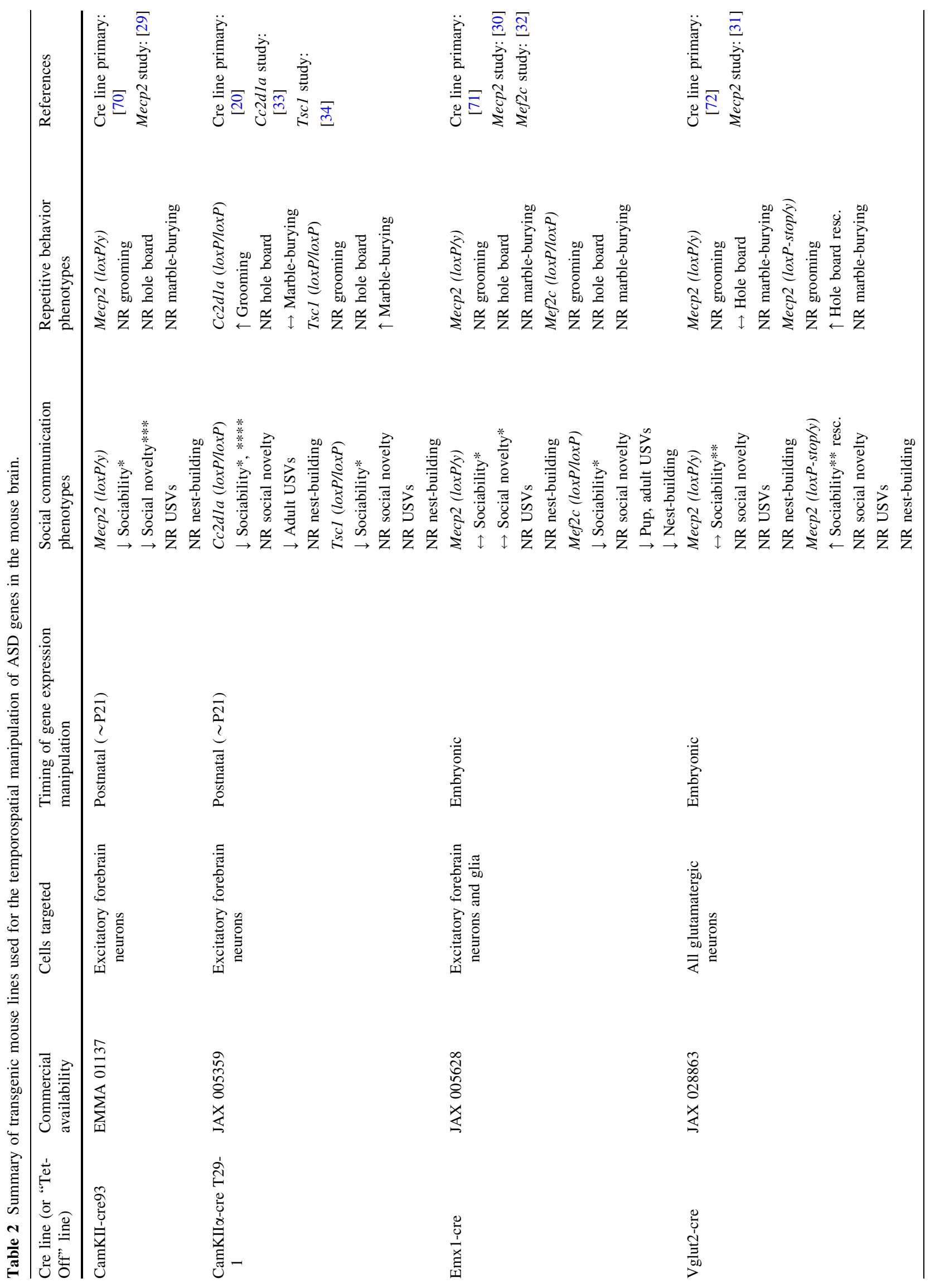




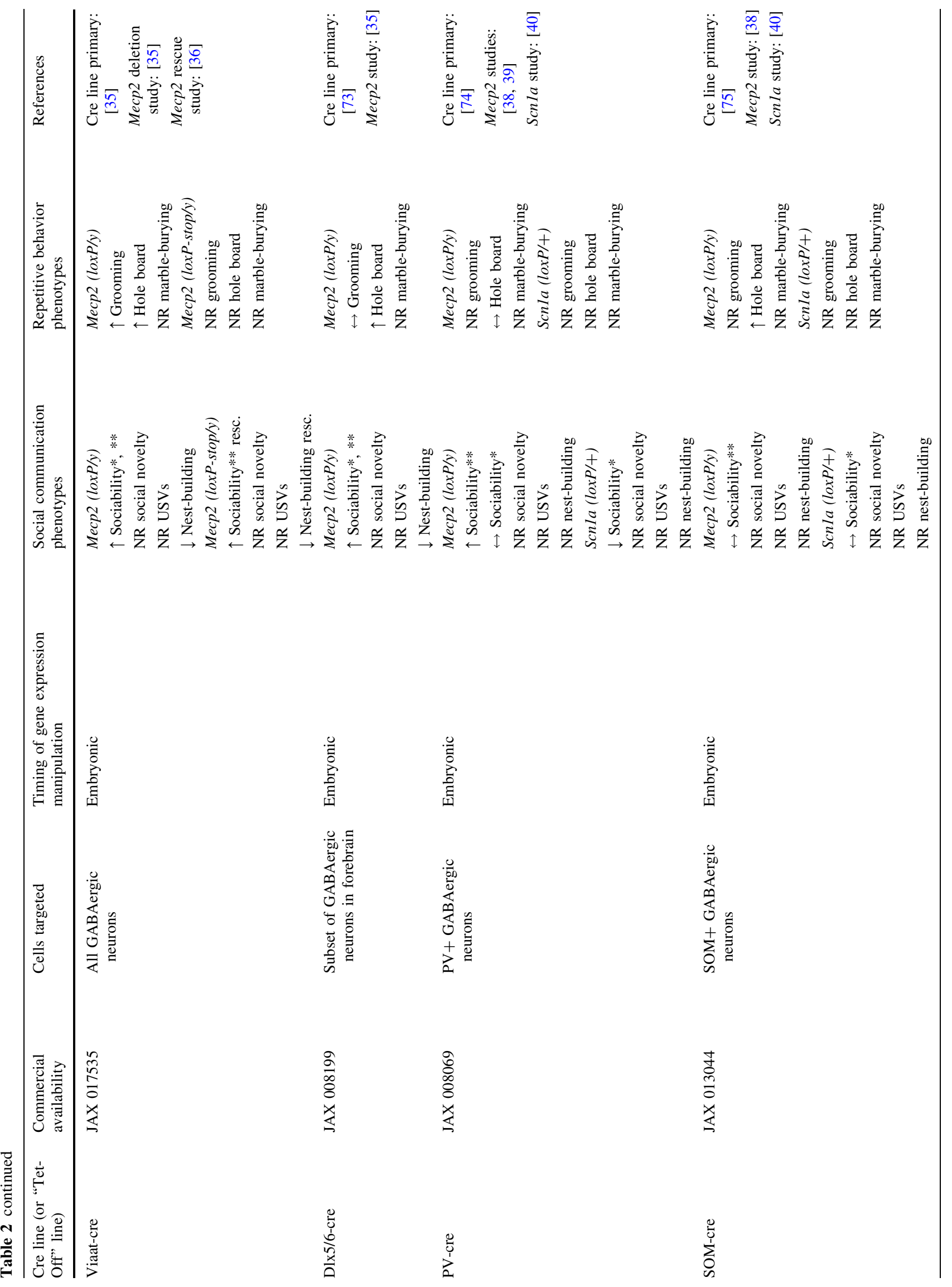




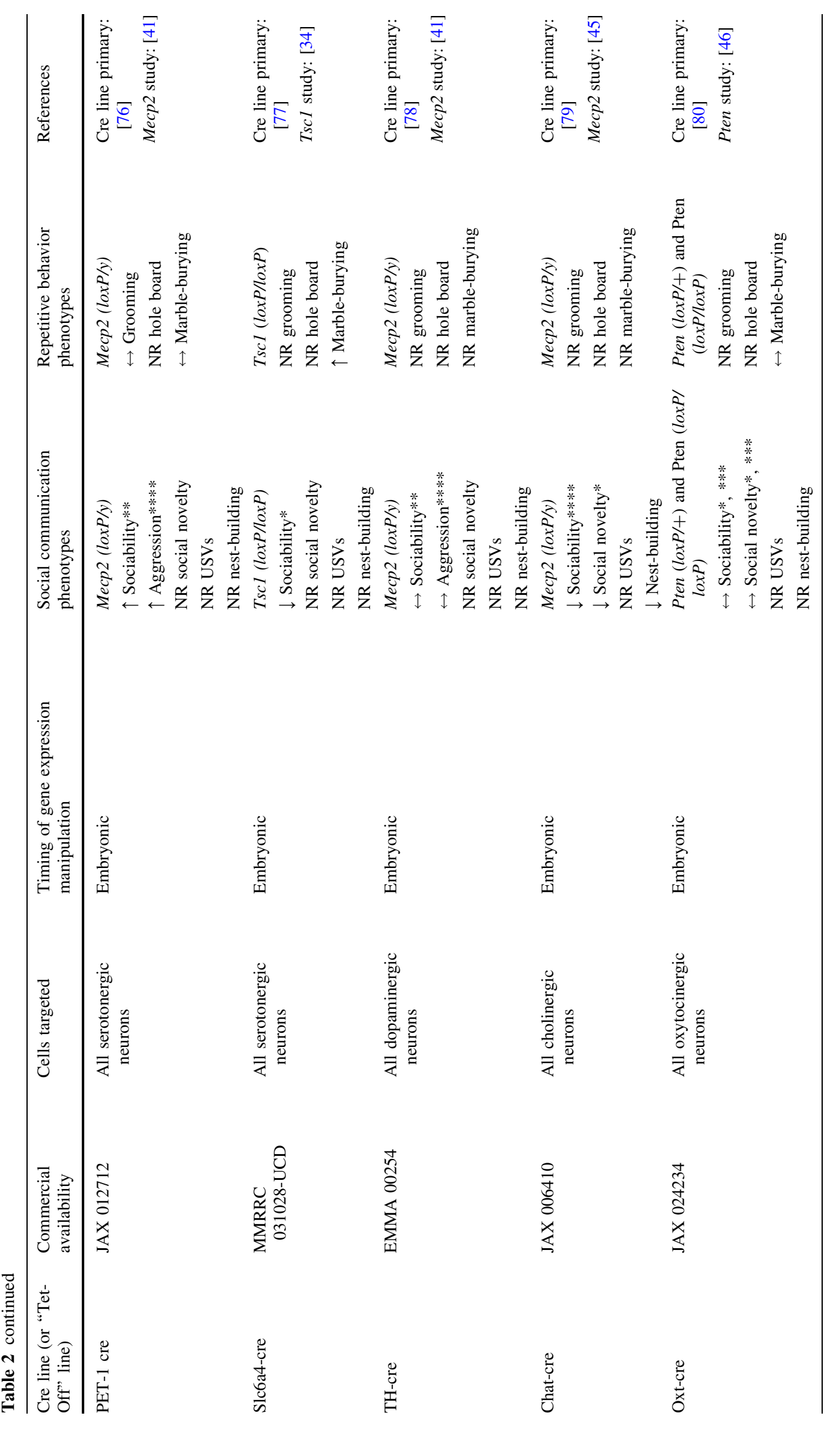




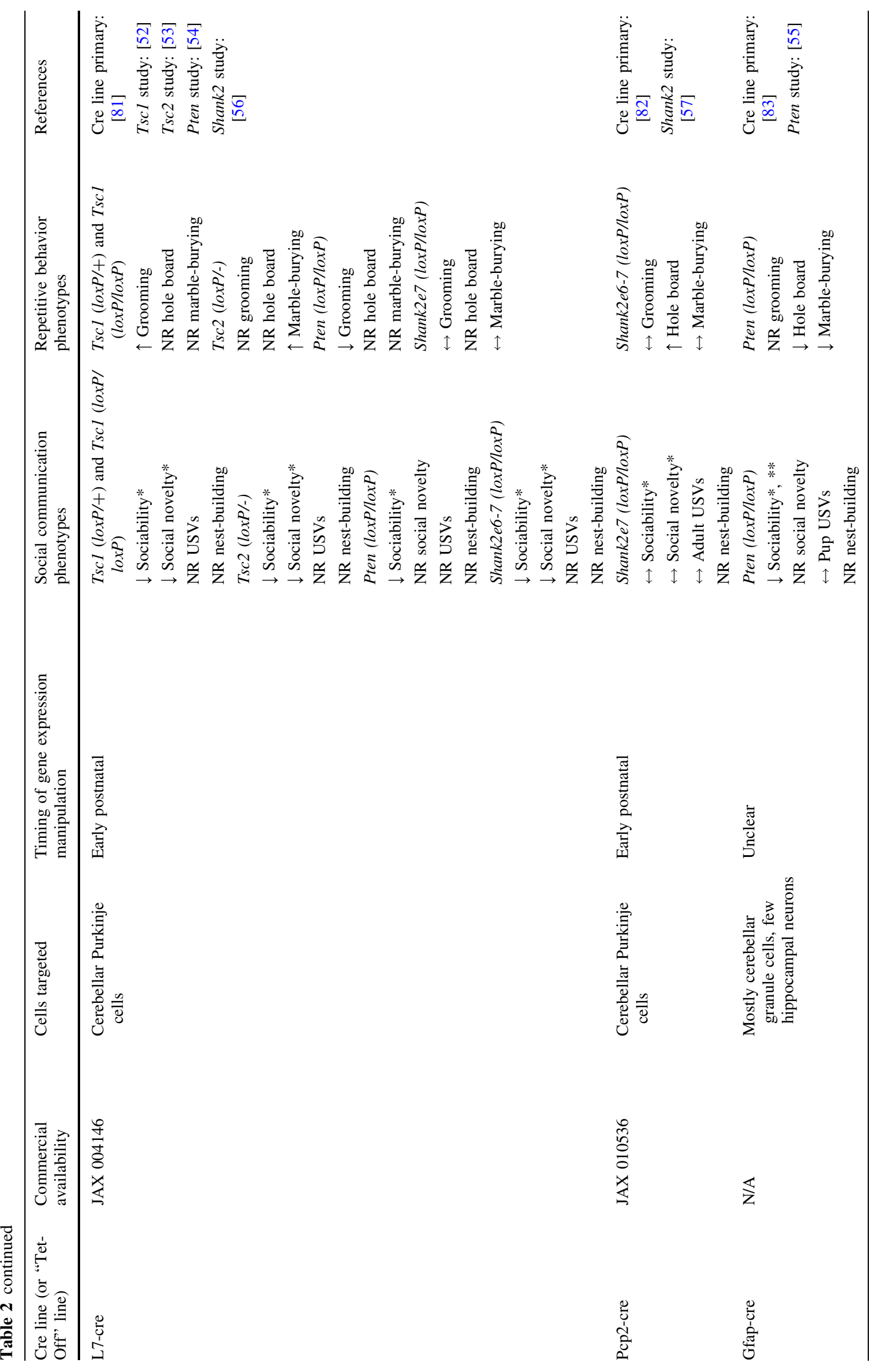




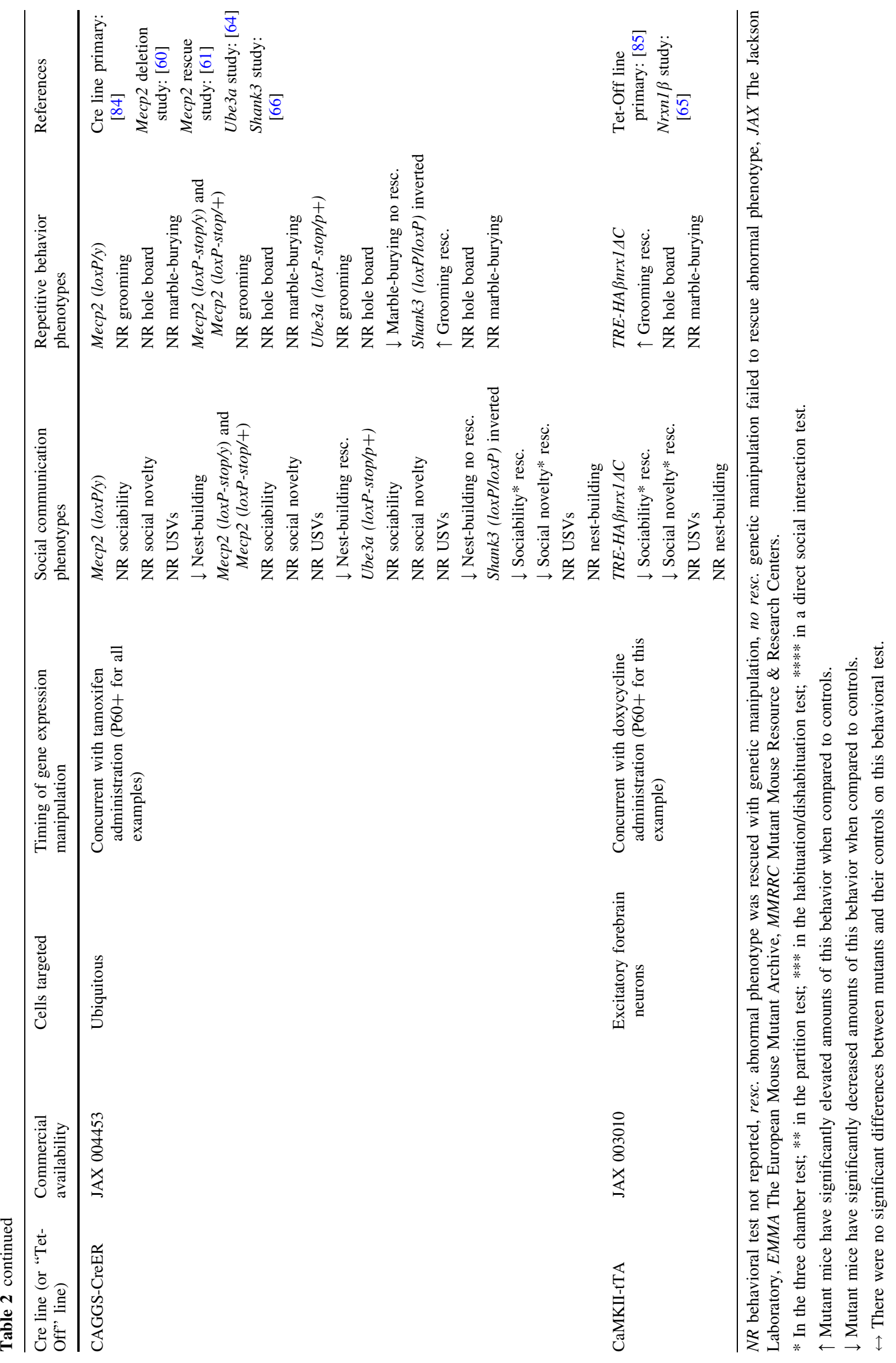


ASDs, research using other models and additional ASDrelevant behaviors is necessary to make more definitive conclusions. An interesting and important question is whether the findings from mouse models can be translated to humans and if the reversibility of symptoms holds true.

\section{Concluding Remarks and Future Directions}

It is clear that, at least in some cases, disrupting the function of either excitatory or inhibitory neurons is sufficient to cause the core behaviors of ASD. Likewise, manipulations of either forebrain or cerebellar neurons can induce ASD-like phenotypes in mice. The study of specific regions outside of the cerebellum is one particularly important direction for future study. For example, several studies have implicated the striatum in the pathophysiology of ASDs by showing region-specific molecular and electrophysiological alterations [67, 68]. Moreover, suppression of Shank3 expression using shRNA targeted to the ventral tegmental area can induce social deficits [69]. However, specific circuits have not yet been causally linked to ASD-relevant behaviors using cell-type specific Cre lines.

The findings reviewed here (summarized in Table 2) support the value of conditional gene-expression technology to delineate the cells and circuits underlying ASDs. However, because these studies are mostly conducted in syndromic ASD models, it remains to be seen whether these findings and conclusions are unique to these specific ASD-causing mutations. Since there are inconsistencies between studies that manipulate the same gene, it may be premature to generalize the limited available evidence. Human genetics studies have supported the role of several hundreds of genes in ASDs, so one challenge is to find out whether there are shared circuit-level mechanisms among ASDs caused by different mutations or etiologies. Caution must be taken when interpreting the results from studies that utilize conditional gene expression. Too often the efficiency and specificity of the Cre lines are overestimated. Moreover, disruption of one set of cells may have downstream or compensatory effects on other cell types in the same brain region, or even in different brain regions due to axonal projections. Hopefully, the development of more precise technology for manipulating cell types and circuits will facilitate a more complete understanding of the neural underpinnings of ASDs.

Acknowledgements We would like to thank the members of the Jiang lab for their contributions to this review. S.W.H. is supported by a Weatherstone Predoctoral Fellowship from Autism Speaks. The research in the Jiang lab is supported by NIH Grants 5R01MH098114-03, 1R21-HD077197-01, and 1R21-MH104316-01, a Duke Institute for Brain Science Incubator award, Autism Speaks, the
Marcus Foundation, the Foundation for Prader-Willi Research, the Phelan-McDermid Syndrome Foundation, the Ruth. K. Broad Foundation, Roche Inc., and SAGE Inc.

Open Access This article is distributed under the terms of the Creative Commons Attribution 4.0 International License (http://crea tivecommons.org/licenses/by/4.0/), which permits unrestricted use, distribution, and reproduction in any medium, provided you give appropriate credit to the original author(s) and the source, provide a link to the Creative Commons license, and indicate if changes were made.

\section{References}

1. Hulbert SW, Jiang YH. Monogenic mouse models of autism spectrum disorders: Common mechanisms and missing links. Neuroscience 2016, 321: 3-23.

2. Alexander AL, Lee JE, Lazar M, Boudos R, DuBray MB, Oakes TR, et al. Diffusion tensor imaging of the corpus callosum in Autism. Neuroimage 2007, 34: 61-73.

3. Di Martino A, Yan CG, Li Q, Denio E, Castellanos FX, Alaerts $\mathrm{K}$, et al. The autism brain imaging data exchange: towards a large-scale evaluation of the intrinsic brain architecture in autism. Mol Psychiatry 2014, 19: 659-667.

4. Sztainberg Y, Zoghbi HY. Lessons learned from studying syndromic autism spectrum disorders. Nat Neurosci 2016, 19: $1408-1417$.

5. American Psychiatric Association. DSM-5 Task Force. Diagnostic and statistical manual of mental disorders: DSM-5. 5th ed. Washington, D.C.: American Psychiatric Association, 2013.

6. Silverman JL, Yang M, Lord C, Crawley JN. Behavioural phenotyping assays for mouse models of autism. Nat Rev Neurosci 2010, 11: 490-502.

7. Bey AL, Jiang YH. Overview of mouse models of autism spectrum disorders. Curr Protoc Pharmacol 2014, 66: 5.66.61-26.

8. Nadler JJ, Moy SS, Dold G, Trang D, Simmons N, Perez A, et al. Automated apparatus for quantitation of social approach behaviors in mice. Genes Brain Behav 2004, 3: 303-314.

9. Choleris E, Little SR, Mong JA, Puram SV, Langer R, Pfaff DW. Microparticle-based delivery of oxytocin receptor antisense DNA in the medial amygdala blocks social recognition in female mice. Proc Natl Acad Sci U S A 2007, 104: 4670-4675.

10. Kudryavtseva NN. Use of the "partition" test in behavioral and pharmacological experiments. Neurosci Behav Physiol 2003, 33 : 461-471.

11. Moretti P, Bouwknecht JA, Teague R, Paylor R, Zoghbi HY. Abnormalities of social interactions and home-cage behavior in a mouse model of Rett syndrome. Hum Mol Genet 2005, 14: 205-220.

12. Terranova ML, Laviola G. Scoring of social interactions and play in mice during adolescence. Curr Protoc Toxicol 2005, Chapter 13: Unit13.10.

13. Winslow JT, Miczek KA. Habituation of aggression in mice: pharmacological evidence of catecholaminergic and serotonergic mediation. Psychopharmacology (Berl) 1983, 81: 286-291.

14. Scattoni ML, Crawley J, Ricceri L. Ultrasonic vocalizations: a tool for behavioural phenotyping of mouse models of neurodevelopmental disorders. Neurosci Biobehav Rev 2009, 33: 508-515.

15. Deacon RM. Assessing nest building in mice. Nat Protoc 2006, 1: 1117-1119.

16. Crawley JN. Translational animal models of autism and neurodevelopmental disorders. Dialogues Clin Neurosci 2012, 14: 293-305. 
17. Moy SS, Nadler JJ, Poe MD, Nonneman RJ, Young NB, Koller $\mathrm{BH}$, et al. Development of a mouse test for repetitive, restricted behaviors: relevance to autism. Behav Brain Res 2008, 188: 178-194.

18. Thomas A, Burant A, Bui N, Graham D, Yuva-Paylor LA, Paylor R. Marble burying reflects a repetitive and perseverative behavior more than novelty-induced anxiety. Psychopharmacology (Berl) 2009, 204: 361-373.

19. Gierut JJ, Jacks TE, Haigis KM. Strategies to achieve conditional gene mutation in mice. Cold Spring Harb Protoc 2014, 2014: 339-349.

20. Tsien JZ, Chen DF, Gerber D, Tom C, Mercer EH, Anderson DJ, et al. Subregion- and cell type-restricted gene knockout in mouse brain. Cell 1996, 87: 1317-1326.

21. Metzger D, Clifford J, Chiba H, Chambon P. Conditional sitespecific recombination in mammalian cells using a ligand-dependent chimeric Cre recombinase. Proc Natl Acad Sci U S A 1995, 92: 6991-6995.

22. Sprengel R, Hasan MT. Tetracycline-controlled genetic switches. Handb Exp Pharmacol 2007: 49-72.

23. Heldt SA, Ressler KJ. The use of lentiviral vectors and Cre/loxP to investigate the function of genes in complex behaviors. Front Mol Neurosci 2009, 2: 22.

24. Semprini S, Troup TJ, Kotelevtseva N, King K, Davis JR, Mullins LJ, et al. Cryptic loxP sites in mammalian genomes: genomewide distribution and relevance for the efficiency of BAC/PAC recombineering techniques. Nucleic Acids Res 2007, 35: $1402-1410$

25. Yin H, Xue W, Chen S, Bogorad RL, Benedetti E, Grompe M, et al. Genome editing with Cas9 in adult mice corrects a disease mutation and phenotype. Nat Biotechnol 2014, 32: 551-553.

26. Wang J, Li X, Zhao Y, Li J, Zhou Q, Liu Z. Generation of celltype-specific gene mutations by expressing the sgRNA of the CRISPR system from the RNA polymerase II promoters. Protein Cell 2015, 6: 689-692.

27. Fu Y, Foden JA, Khayter C, Maeder ML, Reyon D, Joung JK, et al. High-frequency off-target mutagenesis induced by CRISPR-Cas nucleases in human cells. Nat Biotechnol 2013, 31: 822-826.

28. Rubenstein JL, Merzenich MM. Model of autism: increased ratio of excitation/inhibition in key neural systems. Genes Brain Behav 2003, 2: 255-267.

29. Gemelli T, Berton O, Nelson ED, Perrotti LI, Jaenisch R, Monteggia LM. Postnatal loss of methyl-CpG binding protein 2 in the forebrain is sufficient to mediate behavioral aspects of Rett syndrome in mice. Biol Psychiatry 2006, 59: 468-476.

30. Goffin D, Brodkin ES, Blendy JA, Siegel SJ, Zhou Z. Cellular origins of auditory event-related potential deficits in Rett syndrome. Nat Neurosci 2014, 17: 804-806.

31. Meng X, Wang W, Lu H, He LJ, Chen W, Chao ES, et al. Manipulations of $\mathrm{MeCP} 2$ in glutamatergic neurons highlight their contributions to Rett and other neurological disorders. Elife 2016, 5.

32. Harrington AJ, Raissi A, Rajkovich K, Berto S, Kumar J, Molinaro $\mathrm{G}$, et al. MEF2C regulates cortical inhibitory and excitatory synapses and behaviors relevant to neurodevelopmental disorders. Elife 2016, 5

33. Oaks AW, Zamarbide M, Tambunan DE, Santini E, Di Costanzo $\mathrm{S}$, Pond HL, et al. Cc2d1a loss of function disrupts functional and morphological development in forebrain neurons leading to cognitive and social deficits. Cereb Cortex 2016. doi:10.1093/ cercor/bhw009.

34. McMahon JJ, Yu W, Yang J, Feng H, Helm M, McMahon E, et al. Seizure-dependent mTOR activation in 5-HT neurons promotes autism-like behaviors in mice. Neurobiol Dis 2015, 73: 296-306.
35. Chao HT, Chen H, Samaco RC, Xue M, Chahrour M, Yoo J, et al. Dysfunction in GABA signalling mediates autism-like stereotypies and Rett syndrome phenotypes. Nature 2010, 468: 263-269.

36. Ure $\mathrm{K}, \mathrm{Lu} \mathrm{H}$, Wang $\mathrm{W}$, Ito-Ishida $\mathrm{A}, \mathrm{Wu} \mathrm{Z}, \mathrm{He} \mathrm{LJ}$, et al. Restoration of Mecp2 expression in GABAergic neurons is sufficient to rescue multiple disease features in a mouse model of Rett syndrome. Elife 2016, 5.

37. Rudy B, Fishell G, Lee S, Hjerling-Leffler J. Three groups of interneurons account for nearly $100 \%$ of neocortical GABAergic neurons. Dev Neurobiol 2011, 71: 45-61.

38. Ito-Ishida A, Ure K, Chen H, Swann JW, Zoghbi HY. Loss of $\mathrm{MeCP} 2$ in parvalbumin-and somatostatin-expressing neurons in mice leads to distinct Rett syndrome-like phenotypes. Neuron 2015, 88: 651-658.

39. He LJ, Liu N, Cheng TL, Chen XJ, Li YD, Shu YS, et al. Conditional deletion of Mecp2 in parvalbumin-expressing GABAergic cells results in the absence of critical period plasticity. Nat Commun 2014, 5: 5036.

40. Rubinstein M, Han S, Tai C, Westenbroek RE, Hunker A, Scheuer T, et al. Dissecting the phenotypes of Dravet syndrome by gene deletion. Brain 2015, 138: 2219-2233.

41. Samaco RC, Mandel-Brehm C, Chao HT, Ward CS, FyffeMaricich SL, Ren J, et al. Loss of $\mathrm{MeCP} 2$ in aminergic neurons causes cell-autonomous defects in neurotransmitter synthesis and specific behavioral abnormalities. Proc Natl Acad Sci U S A 2009, 106: 21966-21971.

42. Riday TT, Dankoski EC, Krouse MC, Fish EW, Walsh PL, Han JE, et al. Pathway-specific dopaminergic deficits in a mouse model of Angelman syndrome. J Clin Invest 2012, 122: 4544-4554.

43. Mulherkar SA, Jana NR. Loss of dopaminergic neurons and resulting behavioural deficits in mouse model of Angelman syndrome. Neurobiol Dis 2010, 40: 586-592.

44. Rothwell PE, Fuccillo MV, Maxeiner S, Hayton SJ, Gokce O, Lim BK, et al. Autism-associated neuroligin-3 mutations commonly impair striatal circuits to boost repetitive behaviors. Cell 2014, 158: 198-212.

45. Zhang Y, Cao SX, Sun P, He HY, Yang CH, Chen XJ, et al. Loss of MeCP2 in cholinergic neurons causes part of RTT-like phenotypes via $\alpha 7$ receptor in hippocampus. Cell Res 2016, 26: 728-742.

46. Clipperton-Allen AE, Chen Y, Page DT. Autism-relevant behaviors are minimally impacted by conditional deletion of Pten in oxytocinergic neurons. Autism Res 2016, 9: 1248-1262.

47. Ferguson JN, Young LJ, Hearn EF, Matzuk MM, Insel TR, Winslow JT. Social amnesia in mice lacking the oxytocin gene. Nat Genet 2000, 25: 284-288.

48. Pobbe RL, Pearson BL, Defensor EB, Bolivar VJ, Young WS, Lee HJ, et al. Oxytocin receptor knockout mice display deficits in the expression of autism-related behaviors. Horm Behav 2012, 61: 436-444.

49. Lazzari VM, Becker RO, de Azevedo MS, Morris M, Rigatto K, Almeida S, et al. Oxytocin modulates social interaction but is not essential for sexual behavior in male mice. Behav Brain Res 2013, 244: 130-136.

50. Clipperton-Allen AE, Page DT. Pten haploinsufficient mice show broad brain overgrowth but selective impairments in autism-relevant behavioral tests. Hum Mol Genet 2014, 23: 3490-3505.

51. Mittleman G, Goldowitz D, Heck DH, Blaha CD. Cerebellar modulation of frontal cortex dopamine efflux in mice: relevance to autism and schizophrenia. Synapse 2008, 62: 544-550.

52. Tsai PT, Hull C, Chu Y, Greene-Colozzi E, Sadowski AR, Leech $\mathrm{JM}$, et al. Autistic-like behaviour and cerebellar dysfunction in Purkinje cell Tsc1 mutant mice. Nature 2012, 488: 647-651.

53. Reith RM, McKenna J, Wu H, Hashmi SS, Cho SH, Dash PK, et al. Loss of Tsc2 in Purkinje cells is associated with autistic-like 
behavior in a mouse model of tuberous sclerosis complex. Neurobiol Dis 2013, 51: 93-103.

54. Cupolillo D, Hoxha E, Faralli A, De Luca A, Rossi F, Tempia F, et al. Autistic-Like Traits and Cerebellar Dysfunction in Purkinje Cell PTEN Knock-Out Mice. Neuropsychopharmacology 2016, 41: 1457-1466.

55. Lugo JN, Smith GD, Arbuckle EP, White J, Holley AJ, Floruta $\mathrm{CM}$, et al. Deletion of PTEN produces autism-like behavioral deficits and alterations in synaptic proteins. Front Mol Neurosci 2014, 7: 27.

56. Peter S, Ten Brinke MM, Stedehouder J, Reinelt CM, Wu B, Zhou H, et al. Dysfunctional cerebellar Purkinje cells contribute to autism-like behaviour in Shank2-deficient mice. Nat Commun 2016, 7: 12627.

57. Ha S, Lee D, Cho YS, Chung C, Yoo YE, Kim J, et al. Cerebellar Shank2 regulates excitatory synapse density, motor coordination, and specific repetitive and anxiety-like behaviors. J Neurosci 2016, 36: 12129-12143.

58. Jiang YH, Ehlers MD. Modeling autism by SHANK gene mutations in mice. Neuron 2013, 78: 8-27.

59. Berger JM, Rohn TT, Oxford JT. Autism as the Early Closure of a Neuroplastic Critical Period Normally Seen in Adolescence. Biol Syst Open Access 2013, 1.

60. McGraw CM, Samaco RC, Zoghbi HY. Adult neural function requires MeCP2. Science 2011, 333: 186.

61. Lang M, Wither RG, Colic S, Wu C, Monnier PP, Bardakjian BL, et al. Rescue of behavioral and EEG deficits in male and female Mecp2-deficient mice by delayed Mecp2 gene reactivation. Hum Mol Genet 2014, 23: 303-318.

62. Guy J, Gan J, Selfridge J, Cobb S, Bird A. Reversal of neurological defects in a mouse model of Rett syndrome. Science 2007, 315: 1143-1147.

63. Robinson L, Guy J, McKay L, Brockett E, Spike RC, Selfridge J, et al. Morphological and functional reversal of phenotypes in a mouse model of Rett syndrome. Brain 2012, 135: 2699-2710.

64. Silva-Santos S, van Woerden GM, Bruinsma CF, Mientjes E, Jolfaei MA, Distel B, et al. Ube3a reinstatement identifies distinct developmental windows in a murine Angelman syndrome model. J Clin Invest 2015, 125: 2069-2076.

65. Rabaneda LG, Robles-Lanuza E, Nieto-González JL, Scholl FG. Neurexin dysfunction in adult neurons results in autistic-like behavior in mice. Cell Rep 2014, 8: 338-346.

66. Mei Y, Monteiro P, Zhou Y, Kim JA, Gao X, Fu Z, et al. Adult restoration of Shank3 expression rescues selective autistic-like phenotypes. Nature 2016, 530: 481-484.

67. Fuccillo MV. Striatal circuits as a common node for autism pathophysiology. Front Neurosci 2016, 10: 27.

68. Wang X, Bey AL, Katz BM, Badea A, Kim N, David LK, et al. Altered mGluR5-Homer scaffolds and corticostriatal connectivity in a Shank3 complete knockout model of autism. Nat Commun 2016, 7: 11459.

69. Bariselli S, Tzanoulinou S, Glangetas C, Prévost-Solié C, Pucci L, Viguié J, et al. SHANK3 controls maturation of social reward circuits in the VTA. Nat Neurosci 2016, 19: 926-934.
70. Minichiello L, Korte M, Wolfer D, Kühn R, Unsicker K, Cestari $\mathrm{V}$, et al. Essential role for TrkB receptors in hippocampus-mediated learning. Neuron 1999, 24: 401-414.

71. Gorski JA, Talley T, Qiu M, Puelles L, Rubenstein JL, Jones KR. Cortical excitatory neurons and glia, but not GABAergic neurons, are produced in the Emx1-expressing lineage. J Neurosci 2002, 22: 6309-6314.

72. Vong L, Ye C, Yang Z, Choi B, Chua S, Lowell BB. Leptin action on GABAergic neurons prevents obesity and reduces inhibitory tone to POMC neurons. Neuron 2011, 71: 142-154.

73. Monory K, Massa F, Egertová M, Eder M, Blaudzun H, Westenbroek R, et al. The endocannabinoid system controls key epileptogenic circuits in the hippocampus. Neuron 2006, 51: $455-466$.

74. Hippenmeyer S, Vrieseling E, Sigrist M, Portmann T, Laengle C, Ladle DR, et al. A developmental switch in the response of DRG neurons to ETS transcription factor signaling. PLoS Biol 2005, 3: e159.

75. Taniguchi $\mathrm{H}, \mathrm{He} \mathrm{M}, \mathrm{Wu} \mathrm{P}, \mathrm{Kim} \mathrm{S}$, Paik R, Sugino K, et al. A resource of Cre driver lines for genetic targeting of GABAergic neurons in cerebral cortex. Neuron 2011, 71: 995-1013.

76. Scott MM, Wylie CJ, Lerch JK, Murphy R, Lobur K, Herlitze S, et al. A genetic approach to access serotonin neurons for in vivo and in vitro studies. Proc Natl Acad Sci U S A 2005, 102: 16472-16477.

77. Gong S, Doughty M, Harbaugh CR, Cummins A, Hatten ME, Heintz $\mathrm{N}$, et al. Targeting Cre recombinase to specific neuron populations with bacterial artificial chromosome constructs. J Neurosci 2007, 27: 9817-9823.

78. Lindeberg J, Usoskin D, Bengtsson H, Gustafsson A, Kylberg A, Söderström S, et al. Transgenic expression of Cre recombinase from the tyrosine hydroxylase locus. Genesis 2004, 40: 67-73.

79. Rossi J, Balthasar N, Olson D, Scott M, Berglund E, Lee CE, et al. Melanocortin-4 receptors expressed by cholinergic neurons regulate energy balance and glucose homeostasis. Cell Metab 2011, 13: 195-204.

80. Wu Z, Xu Y, Zhu Y, Sutton AK, Zhao R, Lowell BB, et al. An obligate role of oxytocin neurons in diet induced energy expenditure. PLoS One 2012, 7: e45167.

81. Barski JJ, Dethleffsen K, Meyer M. Cre recombinase expression in cerebellar Purkinje cells. Genesis 2000, 28: 93-98.

82. Zhang XM, Ng AH, Tanner JA, Wu WT, Copeland NG, Jenkins NA, et al. Highly restricted expression of Cre recombinase in cerebellar Purkinje cells. Genesis 2004, 40: 45-51.

83. Kwon CH, Zhu X, Zhang J, Knoop LL, Tharp R, Smeyne RJ, et al. Pten regulates neuronal soma size: a mouse model of Lhermitte-Duclos disease. Nat Genet 2001, 29: 404-411.

84. Hayashi S, McMahon AP. Efficient recombination in diverse tissues by a tamoxifen-inducible form of Cre: a tool for temporally regulated gene activation/inactivation in the mouse. Dev Biol 2002, 244: 305-318.

85. Mayford M, Bach ME, Huang YY, Wang L, Hawkins RD, Kandel ER. Control of memory formation through regulated expression of a CaMKII transgene. Science 1996, 274: 1678-1683. 